\title{
Qualidade do aprendizado de lesões elementares de pele obtido por meio de duas diferentes estratégias didáticas
}

\author{
Quality of learning of elemental skin lesions obtained through two different didactic \\ strategies
Calidad del aprendizaje de lesiones elementales de piel obtenida por medio de dos diferentes estrategias didácticas

\begin{abstract}
Bruna Maria da Cruz Crescente ${ }^{1}$, Matheus Albert de Souza Puerro², Lorena Fecury Tavares ${ }^{2 *}$, Keise Bastos Cardoso², Caio Vinicius Botelho Brito ${ }^{3}$, Márcia Bitar Portella1.
\end{abstract}

\begin{abstract}
RESUMO
Objetivo: Comparar o aprendizado de lesões elementares de pele por duas diferentes estratégias por alunos de graduação em medicina. Métodos: Foram formados dois grupos de voluntários. Um grupo foi denominado grupo aula e era composto por 21 alunos e o outro grupo foi chamado grupo computador e era composto por 25 alunos. Excluídos os que declararam ter conhecimentos prévios sobre o tema, restaram 20 alunos no grupo aula e 22 no grupo computador. Ambos receberam o mesmo conteúdo de morfologia básica de lesões de pele, diferindo apenas que o grupo aula assistiu uma aula teórica sobre o tema e o grupo computador teve acesso a um roteiro em Powerpoint, acessado individualmente pelos participantes. Ao término das intervenções, os dois grupos fizeram a mesma avaliação e os resultados foram analisados estatisticamente e comparados. Resultados: Não houve superioridade do ensino baseado no computador em relação a métodos tradicionais. Isto indica a necessidade de novas pesquisas para que se possa potencializar ainda mais os benefícios que podem ser obtidos no aprendizado através do computador. Conclusão: Não houve diferença estatisticamente significante nas médias obtidas pelos dois grupos na avaliação, indicando que as duas metodologias foram igualmente eficazes para o aprendizado de lesões elementares de pele.
\end{abstract}

Palavras-chave: Ensino baseado no computador, Aula teórica, Dermatologia, Educação médica, Ensino.

\begin{abstract}
Objective: To compare the learning of elemental skin lesions by two different strategies by undergraduate medical students. Methods: Two groups of volunteers were formed. One group was named group class and was composed of 21 students and the other group was called computer group and was composed of 25 students. Excluding those who reported previous knowledge about the subject, there were 20 students in the class group and 22 in the computer group. Both received the same basic morphology content of skin lesions, differing only that the class group attended a theoretical class on the subject and the computer group had access to a script in Powerpoint, accessed individually by the participants. At the end of the interventions, the two groups made the same evaluation and the results were analyzed statistically and compared. Results: There was no superiority of computer-based teaching in relation to traditional methods. This indicates the need for further research to further enhance the benefits that can be gained through computer learning. Conclusion: There was no statistically significant difference in the means obtained by the two groups in the evaluation, indicating that both methodologies were equally effective for the learning of elemental skin lesions.
\end{abstract}

Keywords: Computer-based teaching, Theoretical classes, Dermatology, Medical education, Teaching.

\footnotetext{
${ }^{1}$ Universidade do Estado do Pará, Programa de Mestrado Ensino em Saúde na Amazônia, Belém- PA, Brasil

2 Faculdade Metropolitana da Amazônia, Curso de Medicina, Belém-PA, Brasil. * E-mail: lorenafecury@gmail.com

3 Universidade do Estado do Pará, Departamento de Saúde Coletiva, Belém-PA, Brasil
} 


\section{RESUMEN}

Objetivo: Comparar el aprendizaje de lesiones elementales de piel por dos diferentes estrategias por alumnos de graduación en medicina. Métodos: Se formaron dos grupos de voluntarios. Un grupo fue llamado grupo de clases y estaba compuesto por 21 alumnos y el otro grupo fue llamado grupo de ordenador y consta de 25 alumnos. Excluidos los que declararon tener conocimientos previos sobre el tema, quedaron 20 alumnos en el grupo aula y 22 en el grupo ordenador. Ambos recibieron el mismo contenido de morfología básica de lesiones de piel, diferenciando sólo que el grupo aula asistió a una clase teórica sobre el tema y el grupo computador tuvo acceso a un itinerario en Powerpoint, accesado individualmente por los participantes. Al término de las intervenciones, los dos grupos hicieron la misma evaluación y los resultados fueron analizados estadísticamente y comparados. Resultados: No hubo superioridad de la enseñanza basada en la computadora en relación a métodos tradicionales. Esto indica la necesidad de nuevas investigaciones para que se puedan potenciar aún más los beneficios que pueden obtenerse en el aprendizaje a través del ordenador. Conclusión: No hubo diferencia estadísticamente significativa en las medias obtenidas por los dos grupos en la evaluación, indicando que las dos metodologías fueron igualmente eficaces para el aprendizaje de lesiones elementales de piel.

Palabras-clave: Enseñanza basada en la computadora, Clase teórica, Dermatología, Educación médica, Enseñanza.

\section{INTRODUÇÃO}

As aulas teóricas têm sido o modelo predominante de ensino nas universidades desde que elas foram fundadas na Europa Ocidental há mais de 900 anos. Entretanto, estudos nas mais diversas áreas de ensino têm mostrado superioridade das metodologias ativas de ensino sobre métodos tradicionais, nos quais estudantes são exclusivamente receptores de informações (FREEMAN et al., 2014).

Entre as metodologias ativas estão abordagens centradas no aluno como o aprendizado baseado em problemas, os grupos de aprendizado cooperativo ou colaborativo de vários tipos, a peer-instruction ou instrução pelos colegas, estratégias de mudança conceitual e o aprendizado através de tecnologias, esse último termo com frequência usado como sinônimo de e-learning (MICHAEL, 2006).

As Instituições de Ensino Superior (IES) têm sido estimuladas a refletir acerca das mudanças no processo de educação para que possam reconhecer seu papel social, enfrentar seus desafios - como o de romper com estruturas cristalizadas e modelos de ensino tradicionais - e formar profissionais de saúde com competências que Ihes permitam recuperar a dimensão essencial do cuidado (SOUZA et al., 2014).

Nessa nova realidade, observada em vários países, surgiram propostas de aprendizado de dermatologia por meio de programas de computador conectados ou não à internet, chamado de aprendizado baseado no computador (computer based learning ou e-learning). Seria uma alternativa ao tempo em ambulatório e sala de aula, com o intuito de ofertar desde conteúdo teórico básico até cenários clínicos. A dermatologia é uma especialidade de natureza visual, o uso de recursos digitais tem ajudado na tentativa de ensinar pontos fundamentais dessa área a qualquer médico. Tal fato, tem demonstrado melhoria no desempenho dos alunos, os quais avaliaram como sendo mais eficazes do que aulas teóricas e estudo em livros textos. Além de serem tão úteis quanto estágios clínicos (SINGH et al., 2011; Lüdert et al.,2008; CIPRIANO et al., 2013).

Embora o aprendizado ativo no computador pareça promissor, ele ainda carece de melhores evidências para que seja utilizado como metodologia de ensino em substituição ou combinado a métodos tradicionais. Desse modo, o objetivo do presente estudo é comparar o aprendizado de lesões elementares de pele obtido por meio de método tradicional (aula expositiva) e método ativo (digitalizado autônomo) por estudantes de graduação em medicina. 


\section{MÉTODOS}

Este é um estudo transversal e quantitativo. Foi realizado entre novembro de 2017 e março de 2018 em uma IES privada de Belém (Faculdade Metropolitana da Amazônia - FAMAZ), posteriormente às aprovações da orientadora, do responsável pela instituição e do Comitê de Ética em pesquisa com número do parecer 2698516.

De um total de 43 alunos matriculados em uma turma do primeiro semestre, 25 aceitaram participar e foram alocados no chamado Grupo Computador. De um total de 38 matriculados em outra turma, 21 aceitaram participar e foram alocados no chamado Grupo Aula.

A aceitação veio após serem esclarecidos sobre os objetivos, o método, os riscos e benefícios e através de assinatura de um termo de consentimento livre e esclarecido. A participação dos alunos foi voluntária, os resultados foram usados apenas para finalidade de pesquisa e as notas dos alunos não foram afetadas.

Aos alunos dos dois grupos, solicitou-se que preenchessem a ficha do protocolo do trabalho com nome, sexo, idade, formação superior prévia e se tinham algum conhecimento preexistente sobre o tema a ser ministrado. O objetivo era conhecer as características dos grupos e excluir aqueles que tivessem conhecimento prévio. Foram excluídos 3 alunos no grupo computador e 1 aluno no grupo aula segundo esse critério de exclusão.

O Grupo Computador recebeu o conteúdo de lesões elementares de pele por meio de um roteiro autoexplicativo em powerpoint, inserido no computador no laboratório de informática da instituição, onde existem cabines individuais com computadores. Os alunos deste grupo não puderam tirar dúvidas sobre o conteúdo do roteiro, por se considerar que esta seria uma limitação própria do método.

Ao final do roteiro, o aluno abria o arquivo "Avaliação" e nele observava 10 imagens e, de posse de uma lista com o nome das 22 lesões elementares apresentadas no roteiro, precisava identificar qual lesão elementar era apresentada em cada imagem. Não houve tempo estipulado para realizar a avaliação, tanto no grupo computador quanto no grupo aula.

O Grupo Aula recebeu conteúdo equivalente por meio de aula teórica tradicional em uma sala de aula, utilizando powerpoint e Datashow. No fim da aula realizou a mesma avaliação do Grupo Computador, porém com as imagens projetadas através do datashow. A aula teórica foi ministrada pela pesquisadora (dermatologista e professora da IES) e os alunos puderam tirar dúvidas durante e imediatamente após a aula, mas não durante a avaliação.

Foram utilizadas as mesmas imagens, sem diferença na resolução, no roteiro do computador e na aula teórica. O conteúdo explicativo, que acompanhava as imagens no roteiro do computador, foi falado pela pesquisadora durante a aula teórica para os alunos do Grupo Aula, a diferença foi apenas a forma de obtenção: no Grupo Computador era ativo e no Grupo Aula passivo.

$\mathrm{Na}$ ficha do protocolo do trabalho, ao terminar de realizar a avaliação das 10 imagens, os alunos dos dois grupos foram solicitados a responder se consideraram a atividade que foram submetidos ótima, boa, regular ou ruim e se julgavam que haviam absorvido o conteúdo. Havia ainda um espaço para que realizassem críticas e sugestões, caso desejassem.

As avaliações foram corrigidas ao final, sendo atribuído 1 ponto para cada imagem. Foi ainda atribuído um grau de dificuldade para cada questão entre fácil, moderado e difícil. Estabelecidos esses critérios, 4 imagens foram consideradas como fáceis, 3 como moderadas e 3 como difíceis. Os resultados obtidos pelos alunos dos dois grupos foram contabilizados e comparados estatisticamente.

Os resultados obtidos pelos alunos dos dois grupos foram contabilizados e organizados utilizando o programa Excel 2017. Para a análise descritiva dos dados, foram confeccionados gráficos e tabelas utilizando o mesmo programa. Para as análises de correlação dos dados foram aplicados estudos de qui-quadrado independência, teste $g$-independência, $t$-student independência, sendo considerado o $p$ valor $<0,05$ e utilizando o programa Biostat 2008. 


\section{RESULTADOS}

Conhecidos os dois grupos - o grupo aula com 20 alunos e o grupo computador com 22 alunos - comparouse, por meio de análise estatística, as médias obtidas na avaliação pelos dois grupos e conclui-se que não houve diferença estatisticamente significante. Sendo assim, indicando que o ensino através do computador foi igualmente eficaz à aula teórica para o aprendizado de lesões elementares de pele (Tabela 1).

Tabela 1: Comparação das médias obtidas na avaliação pelos dois grupos.

\begin{tabular}{ccccc}
\hline Grupo & Mínimo & Máximo & Média & Desvio Padrão \\
\hline Aula & 2 & 10 & 6.8 & \pm 2.0 \\
Computador & 2 & 9 & 6.2 & \pm 1.7 \\
Geral & 2 & 10 & 6.5 & \pm 1.8 \\
\hline
\end{tabular}

$p=0.1443$ Teste t Student Independência

Fonte: Pesquisa de campo, Belém, 2018.

Separou-se ainda, um grupo dos que tinham formação prévia e observou-se a mesma média dos que não tinham, reforçando não haver necessidade de excluir esses participantes (Tabela 2).

Tabela 2: Média de acertos entre alunos com ou sem formação prévia.

\begin{tabular}{cccccc}
\hline Grupo & \multicolumn{2}{c}{ Com formação prévia } & \multicolumn{2}{c}{ Sem formação prévia } & p-valor \\
\cline { 2 - 4 } Aula & Média & DP & Média & DP & \\
Computador & 6.9 & \pm 1.9 & 6.8 & \pm 2.2 & 0.4644 \\
Geral & 6.5 & \pm 3.5 & 6.2 & \pm 1.6 & 0.3949 \\
& 6.8 & \pm 2.0 & 6.4 & \pm 1.8 & 0.2951
\end{tabular}

Fonte: Pesquisa de campo, Belém, 2018.

Não houve tampouco, diferença significante nas notas obtidas pelos alunos, ao se considerar a distribuição das mesmas, com porcentagens equivalentes de notas entre 2 e 4, entre 5 e 7 e entre 8 e 10 nos dois grupos. A menor nota obtida foi 2 em ambos os grupos e a maior foi 9 no grupo computador e 10 no grupo aula (Tabela 3).

Tabela 3: Notas dos grupos na avaliação, distribuídas por intervalos.

\begin{tabular}{|c|c|c|c|c|c|c|}
\hline \multirow{2}{*}{$\begin{array}{c}\text { Nota na Avaliação } \\
02 \text { a } 04\end{array}$} & \multicolumn{2}{|c|}{ Grupo Aula } & \multicolumn{2}{|c|}{ Grupo Computador } & \multicolumn{2}{|c|}{ Geral } \\
\hline & 2 & $10.0 \%$ & 3 & $13.6 \%$ & 5 & $11.9 \%$ \\
\hline 05 a 07 & 11 & $55.0 \%$ & 15 & $68.2 \%$ & 26 & $61.9 \%$ \\
\hline 08 a 10 & 7 & $35.0 \%$ & 4 & $18.2 \%$ & 11 & $26.2 \%$ \\
\hline Mín - Máx & \multicolumn{2}{|c|}{$02-10$} & \multicolumn{2}{|c|}{$02-09$} & \multicolumn{2}{|c|}{$02-10$} \\
\hline Média \pm DP & \multicolumn{2}{|c|}{$6.8 \pm 2.0$} & \multicolumn{2}{|c|}{$6.2 \pm 1.7$} & \multicolumn{2}{|c|}{$6.5 \pm 1.8$} \\
\hline
\end{tabular}

$\mathbf{p}=\mathbf{0 . 4 8 6 3}$ Teste Qui-Quadrado Independência

Fonte: Pesquisa de campo, Belém, 2018. 
Além das notas gerais de 1 a 10, instituiu-se uma gradação de dificuldade para as questões. Os alunos dos dois grupos tiveram a mesma média de acertos nos três tipos de questões. Acertaram, na média 3 , das 4 questões fáceis; 2, das 3 questões moderadas; e 2, das 3 questões difíceis. Assim sendo, os alunos dos dois grupos tiveram não só a mesma média de acertos, mas também a mesma habilidade para detectar as características de cada lesão. Mais um ponto que indica a igual eficácia de aprendizado dos dois métodos (Tabela 4).

Tabela 4: Média de acertos por grau de dificuldade.

\begin{tabular}{cccccccc}
\hline \multirow{2}{*}{ Questões } & N & \multicolumn{2}{c}{ Grupo Aula } & \multicolumn{2}{c}{ Grupo Computador } & \multicolumn{2}{c}{ Geral } \\
\cline { 3 - 8 } & & Média & DP & Média & DP & Média & DP \\
Fáceis & $\mathbf{4}$ & 3 & \pm 0.7 & 3 & \pm 0.9 & $\mathbf{3}$ & \pm 0.8 \\
Moderadas & $\mathbf{3}$ & 2 & \pm 0.8 & 2 & \pm 0.7 & $\mathbf{2}$ & \pm 0.7 \\
Difíceis & $\mathbf{3}$ & 2 & \pm 0.9 & 2 & \pm 0.9 & $\mathbf{2}$ & \pm 0.9 \\
\hline
\end{tabular}

Fonte: Pesquisa de campo, Belém, 2018.

Um tópico que foi solicitado aos alunos que preenchessem no protocolo foi a autopercepção de absorção do conteúdo. Apenas 1 aluno do grupo aula declarou não ter absorvido o conteúdo, enquanto no grupo computador foram 5 alunos. Esse dado não mostrou diferença estatisticamente significante. Como essa percepção é subjetiva, foi analisada a comparação da percepção do aluno com o seu desempenho na avaliação. Não foi possível fazer essa comparação no grupo aula, pois só um aluno declarou não ter absorvido o conteúdo. Já no grupo computador, encontrou-se que mesmo os alunos que declararam não ter absorvido o conteúdo obtiveram, na avaliação, a mesma média dos que julgavam ter absorvido, não tendo sido, portanto, uma percepção real (Tabela 5).

Tabela 5: Comparação das médias entre os alunos que declararam, ou não, absorção do conteúdo.

\begin{tabular}{cccccc}
\hline Grupo & \multicolumn{2}{c}{ Relataram absorção } & \multicolumn{2}{c}{ Não relataram absorção } & \multirow{2}{*}{ p-valor } \\
\cline { 2 - 4 } & Média & DP & Média & DP & \\
Aula & 6.8 & \pm 1.9 & 7.0 & ---- & --- \\
Computador & 6.2 & \pm 1.9 & 6.0 & \pm 1.0 & 0.3970 \\
GERAL & 6.5 & \pm 2.0 & 6.2 & \pm 2.0 & 0.3330 \\
\hline
\end{tabular}

Fonte: Pesquisa de campo, Belém, 2018.

\section{DISCUSSÃO}

Os educadores médicos de hoje estão enfrentando desafios diferentes dos de seus antecessores no ensino dos futuros médicos. $O$ ensino tradicional centrado no professor está cedendo lugar a um modelo centrado no aluno, o qual está no controle do seu próprio aprendizado (RUIZ et al., 2006). O uso do computador neste trabalho foi postulado como uma ferramenta educacional colocada a serviço e sob controle de cada aluno.

Esse achado está de acordo com o encontrado por Jenkins et al. (2008), que ao comparar auxílio do computador com aula teórica para o ensino de morfologia básica em dermatologia, não encontrou diferença estatisticamente significante nas notas dos alunos, 4 dias após exposição ao material. Ele concluiu que os mesmos aprendizado e retenção imediata de conhecimento foram obtidos pelas duas metodologias. 
Em nosso trabalho, ao comparar o aprendizado de lesões elementares de pele com o uso do computador ou através de aula teórica não encontramos diferença estatisticamente significativa nas médias dos dois grupos. Esse achado está de acordo com o encontrado por Jenkins et al. (2008), que ao comparar auxílio do computador com aula teórica para o ensino de morfologia básica em dermatologia, não encontraram diferença estatisticamente significante nas notas dos alunos, 4 dias após exposição ao material. Eles concluíram que os mesmos aprendizado e retenção imediata de conhecimento foram obtidos pelas duas metodologias.

Jenkins et al. (2008) afirma que uma das barreiras para a implementação ampla de metodologias com o auxílio do computador é a falta de evidência de que sejam mais eficientes do que outros métodos didáticos.

Chumley-Jones et al. (2002) revisaram a literatura sobre aprendizado baseado no computador acessado via internet (Web-based learning - WBL). Eles observaram que, nos estudos em que o grupo WBL e o grupo controle receberam conteúdo educacional idêntico, foi possível a identificação dos ganhos de aprendizado atribuíveis especificamente ao método web. O mesmo ocorreu no presente trabalho. Os estudos que preenchiam esses critérios demonstraram não haver diferença de ganho de conhecimento entre os grupos. Então, é possível concluir que metodologias computacionais são tão eficientes quanto outros métodos, em concordância com o resultado do presente trabalho.

Berman et al. (2008) apontam para o fato de que os estudos frequentemente são desenhados para responder à pergunta: "O ensino baseado no computador é mais eficiente do que outras metodologias?". Os autores identificam, nessa pergunta, duas suposições incorretas. Essa questão presume que o caminho é usálo em substituição a outras metodologias e que se ele não for melhor, não vale a pena ser usado.

Segundo Fransen et al. (2018), os programas de e-learning estão se tornando populares como um formato educacional adicional na educação médica. Em seu estudo, observaram o aumento significativo na aquisição de conhecimento dermatológico com o programa de e-learning, quando combinado com métodos convencionais de ensino. Entretanto, afirmam que sua eficácia, mesmo que o método seja apreciado pelos estudantes, deve ser rigorosamente avaliada e comparada ao ensino convencional. De acordo com Berman et al. (2008), o uso do computador para melhorar o aprendizado é esperado pela atual geração.

No presente trabalho, observou-se uma tendência a melhor avaliar a aula teórica do que a atividade no computador. No entanto, não houve diferença estatisticamente significante entre a avaliação das duas metodologias.

Nesse trabalho, tomou-se cuidado para que o conteúdo da aula e da atividade no computador fosse idêntico, incluindo as mesmas imagens e explicações. A atividade no computador e a aula teórica foram realizadas em horário além das aulas obrigatórias e de um assunto que eles ainda não usariam naquele semestre, isso pode ter afetado o interesse dos alunos. Além disso, ao iniciar as intervenções, eles não tinham como estimar quanto tempo levariam para terminar, tal fato pode ter gerado impaciência. Na ficha de protocolo, um aluno do grupo computador disse ter achado o tempo curto para a realização da atividade, o que teria afetado a absorção do conteúdo em sua percepção. Porém, havia sido esclarecido que não havia tempo limite.

Já a aula teórica foi ministrada pela própria pesquisadora, o que pode ter influenciado a liberdade dos alunos avaliarem negativamente. Mesmo tendo sido informados que respondessem o que achavam, já que não lhes seria dada nenhuma nota e que estavam avaliando a metodologia e não o desempenho da professora.

Algumas outras hipóteses podem ser levantadas para explicar está tendência de melhor avaliação da aula teórica, diferindo dos resultados de trabalhos anteriores. $O$ assunto ministrado pode ter sido difícil para estudantes do primeiro semestre, que ainda possui muito pouco conhecimento, até mesmo das disciplinas básicas como histologia e patologia, as quais se relacionam com os achados das lesões elementares explicadas na aula teórica. Embora, o tema estivesse explicado também no roteiro do computador, pode não ter ficado tão claro para os alunos que não puderam tirar dúvidas. No trabalho de Cipriano et al. (2013), 94\% dos alunos julgaram que o conteúdo dos módulos online estava claro o suficiente para dispensar a figura de um professor, porém eram alunos do quarto ano de medicina. 
Em alunos igualmente motivados, diferentes métodos podem chegar a um mesmo desempenho de aprendizado, quando comparados (JENKINS et al., 2008). Além disso, a tecnologia em si não é o mais importante. O fundamental é qualidade do material, pois ela impacta o aprendizado. Chumley-Jones et al. (2002) aponta que os educadores precisam reconhecer que programas ou materiais educacionais mal elaborados não são melhorados por serem apresentados em uma página da internet. Da mesma forma, uma aula teórica precisa ser boa, para que impacte o aprendizado e seja bem avaliada.

Neste estudo, os participantes foram expostos ao tema do trabalho pela primeira vez por meio de um método ativo (grupo computador) ou tradicional (grupo aula) e, em seguida, foram avaliados quanto à retenção imediata. No entanto, no ensino tradicional - na maioria das Universidades - a aula teórica é seguida do aprendizado clínico. Acredita-se, então, que o aprendizado clínico poderia suprir as deficiências de ambos os métodos. Contudo, segundo Kumar et al. (2015), o aprendizado clínico também tem seus desafios, entre eles a limitação de tempo e a falta de material clínico adequado.

De fato, durante um período de estágio de um grupo, não há como garantir a exposição a todos os tipos de lesões elementares. Ademais, a realidade de muitos serviços diminui o tempo das consultas, logo, o tempo disponível para que o aluno avalie e aprenda a perceber as características de cada lesão. A não cooperação dos pacientes é outro problema frequentemente encarado quando um grupo de estudantes os examina (KUMAR et al., 2015).

A aula teórica tradicional tem suas vantagens, a principal é a interação em tempo real com o professor, permitindo tirar dúvidas no momento em que surgem (JENKINS et al., 2008).

A atividade no computador parece particularmente promissora na dermatologia, mas a importância deste trabalho está em poder optar por nova metodologia com a segurança de que será útil e de que os alunos não perdem com sua implantação.

Desenvolveu-se nesse trabalho, um programa simples, de baixo custo e sem conexão com a internet. A partir de resultados obtidos que o validam como uma ferramenta útil, será possível elaborar um programa com maior complexidade e com banco de imagens do próprio serviço da IES. Com o auxílio de equipe de tecnologia, pode-se colocar imagens que possam ser avaliadas em zoom, além de associar perguntas e respostas para autoavaliação.

Os programas de computador têm a vantagem de poderem ser revisados, corrigidos e novamente disponibilizados, mais rapidamente e com custo menor do que livros e atlas impressos. Sua desvantagem é que geralmente falta a figura de um editor que revise o conteúdo e a utilidade. Sendo, portanto, interessante que haja uma revisão cuidadosa feita por pares (BERMAN et al., 2008; RUIZ et al., 2006).

Considerando-se os vários pontos das metodologias, observou-se que o aprendizado de lesões elementares não será em um só momento, seja na aula teórica ou no computador. Acredita-se que seja um processo durante o estudo de dermatologia. Pode iniciar na sala de aula ou no computador, é sedimentado no aprendizado dos conceitos com livros e vai se sedimentando na prática clínica e o computador poderá ser uma figura importante também nessa fase pelo que se observou nos estudos. Isso porque as lesões elementares são uma importante representação da habilidade sutil de inspeção, a qual precisa ser desenvolvida. Dessa forma, no final da disciplina ou do estágio o aluno poderá sentir que aprendeu dermatologia e considerar-se-á apto a atender o básico desta especialidade, pois irá se defrontar em sua prática futura.

\section{CONCLUSÃO}

Ao comparar o aprendizado de lesões elementares obtido através de aula teórica ou através de método ativo com auxílio de computador, observou-se neste estudo, igual desempenho dos alunos com as duas metodologias. O uso do computador como ferramenta educacional, seja com ou sem internet (CBL ou elearning), é ainda uma área que pode ser considerada nova, com menos de 30 anos de pesquisas. Há ainda um campo vasto a ser pesquisado nesta área onde os estudos têm, em geral, pequenas amostras e pouca uniformidade para que possam ser comparados. Os alunos esperam poder contar com a tecnologia dos 
computadores como uma ferramenta educacional e cabe aos educadores pesquisar de que forma os benefícios que podem ser obtidos com seu uso, poderão ser ampliados.

\section{REFERÊNCIAS}

1. BERMAN NB, FALL LH, MALONEY CG, et al. Computer-Assisted Instruction in Clinical Education: a Roadmap to increasing CAI Implementation. Advances in Health Sciences Education, 2008; 13: 373-383.

2. CAYCE R, BERGSTRESSER P, HESTERMAN K, et al. Dermatology Curriculum for Internal Medicine Residents: A Randomized Trial. J Grad Med Educ, 2014; 6(2): 296-300.

3. CHUMLEY-JONES HS, DOBBIE A, ALFORD CL, et al. Web-based learning: sound educational method or hype? A review of the evaluation literature. Acad Med, 2002; 77(10): 86-93.

4. CIPRIANO SD, DYBRO E, BOSCARDIN CK, et al. Online learning in a dermatology clerkship: Piloting the new American Academy of Dermatology Medical Student Core Curriculum. J Am Acad Dermatol, 2013; 69(2): 267-72.

5. FRANSEN F, MARTENS H, NAGTZAAM I, et al. Use of e-learning in clinical clerkships: effects on acquisition of dermatological knowledge and learning processes. International Journal of Medical Education, 2018; 9:11-17.

6. FREEMAN S, EDDY SL, McDONOUGH M, et al. Active learning increases student performance in science, engineering and mathematics. Proceedings of the National Academy of Sciences, 2014; 111(23): 8410-8415.

7. HARTMANN AC, CRUZ PD. Interactive mechanisms for teaching dermatology to medical students. Arch Dermatol, 1998; 134: 725-728.

8. JENKINS S, GOEL R, MORREL DS, et al. Computer-assisted instruction versus traditional lecture for medical student teaching of dermatology morphology: A randomized control trial. J Am Acad Dermatol, 2008; 59(2): 255 259.

9. KUMAR G, MADHAVI S, KARTHIKEYAN K, et al. Role of clinical images based teaching as a supplement to conventional teaching in dermatology. Indian Journal of Dermatology, 2015;60: 556.

10. LÜDERT T, NAST A, ZIELKE H, et al. E-learning in the dermatological education at the Charité: evaluation of the last three years. JDDG: Journal der Deutschen dermatologischen Gesellschaft, 2008; 6: 467-472.

11. MICHAEL J. Where's the evidence that active learning works?. Advances in Physiology Education, 2006; 30(4):159-67.

12. RUIZ JG, MINTZER MJ, LEIPZIG RM, et al. The impact of E-learning in medical education. Acad Med, 2006; 81(3): 207-212.

13. SINGH DS, BOUDVILLE N, CORDEROY R, et al. Impact on the dermatology educational experience of medical students with the introduction of online teaching support modules to help address the reduction in clinical teaching. Australasian Journal of Dermatology, 2011; 52: 264-269.

14. SOUZA CS, IGLESIAS AG, PAZIN-FILHO A, et al. Estratégias inovadoras para métodos de ensino tradicionais aspectos gerais. Medicina (Riberão Preto), 2014; 47(3): 284-292.

15. WHINTAKER-WORTH DL, SUSSER WS, GRANT-KELS JM, et al. Clinical dermatologic education and the diagnostic acumen of medical students and primary care residentes. International Journal of Dermatology, 1998; 37: 855-859. 